\title{
Exposure of Engineered Nanoparticles to Human Lung Epithelial Cells: \\ Influence of Chemical Composition and Catalytic Activity on Oxidative Stress
}

Ludwig L. Limbach ${ }^{1}$, Peter Wick², Pius Manser ${ }^{2}$, Robert N. Grass ${ }^{1}$, Arie Bruinink ${ }^{2}$, Wendelin J. Stark ${ }^{1}$

1 Department of Chemistry and Applied biosciences, Institute for Chemical and Bioengineering, ETH Zurich, $\mathrm{CH}-8093 \mathrm{Zurich}$

2 Empa, Swiss Federal Laboratories for Materials Testing and Research, Laboratory for Materials-Biology Interactions, CH-9014 St. Gallen contact: peter.wick@empa.ch

\section{Introduction}

The chemical and catalytic activity of nanoparticles has strongly contributed to the current tremendous interest in engineered nanomaterials and often serves as a guiding principle for the design of functional materials. Since it has most recently become evident that such active materials can enter into cells (Figure 1) or organisms, the present study investigates the level of intracellular oxidations after metal-doped silica nanoparticles and the corresponding pure oxides in vitro. The resulting oxidative stress was quantitatively measured as the release of reactive oxygen species (ROS) by the conversion of the sensitive probe HDCF-DA to the fluorescent DCF (Figure 3).

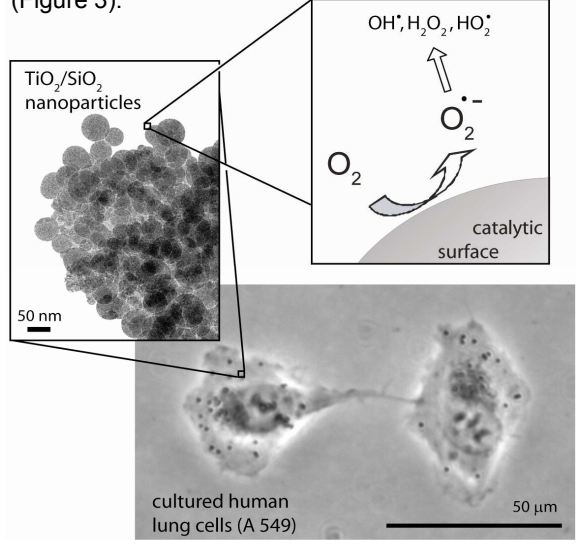

Figure 1: Scheme of catalytic active nanoparticles in human lung epithelial cells

\section{Materials and Methods}

The present study used two sets of materials: (1) silica doped with 0.5 and $1.6 \mathrm{wt} \%$ of iron, cobalt, manganese, and titanium and the corresponding pure oxide; and (2) a series of eight iron-containing silica nanoparticles $\left(0-10 \mathrm{wt} \% \mathrm{Fe} / \mathrm{SiO}_{2}\right)$ to systematically investigate the role of catalytic effects (Figure 2).
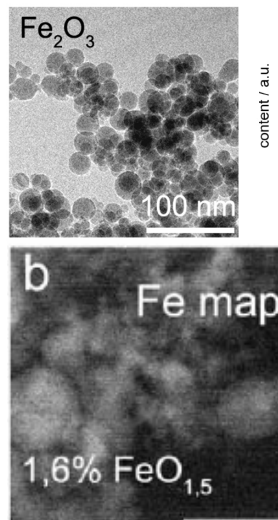
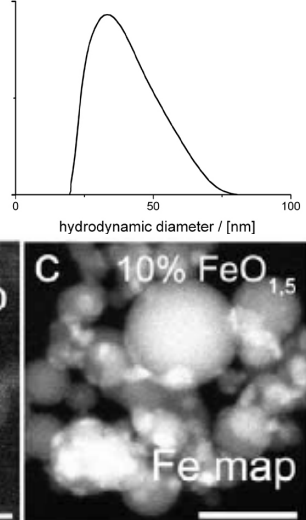

Figure 2: Morphology of applied nanoparticles: (upper Figure) representative transmission electron micrograph of ironoxide and represeative transmission electron micrograph of ironoxide and ray disk centrifugation (b) Scenning transmission eastron micrograph of $1.6 \% \mathrm{FeO}_{15}$ on $\mathrm{SiO}_{2}$ and (c) $10 \% \mathrm{FeO}_{15}$ on $\mathrm{SiO}_{2}$ Size bar $50 \mathrm{~nm}$

\section{Results}
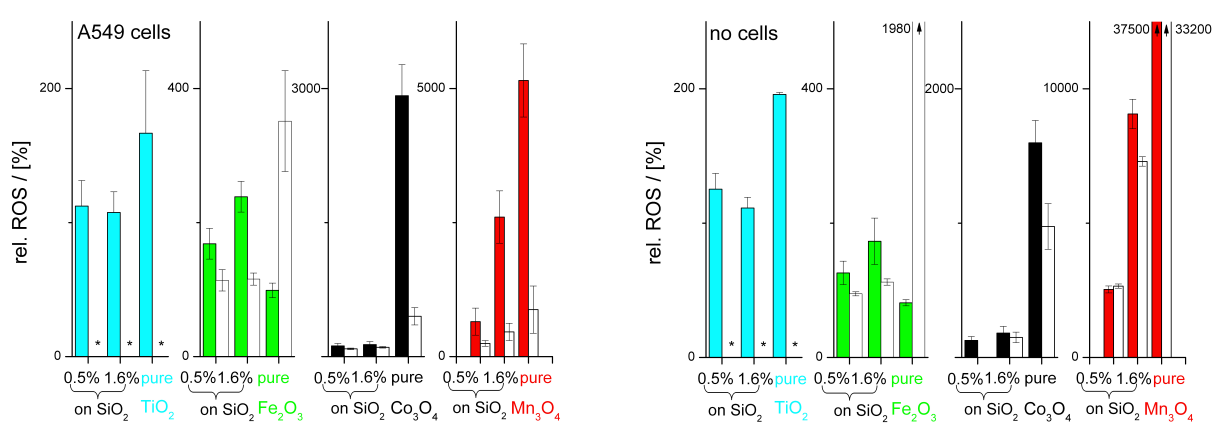

ROS concentration in human lung epithelial cells (left) after $4 \mathrm{~h}$ nanoparticles exposure (full columns) relative to reference cultures without particle exposition. Empty columns depict cultures only exposed to the corresponding amount of metal salts as aqueous solution. Comparison of ROS production in cell free (right) culture medium for heavy metals at $30 \mu \mathrm{g} / \mathrm{ml}$ medium. Exposure to particles (full columns) stimulates few ROS for iron oxide and titania. Dissolved iron ions (empty bars), however, promote a 20 times higher ROS production than exposure to the same amount of iron in the form of $\mathrm{Fe} 2 \mathrm{O} 3$ nanoparticles. This proves that the $\mathrm{FeO} 3$ particles did not significantly dissolve to iron within the duration of the exposure. Note the different scale bares.

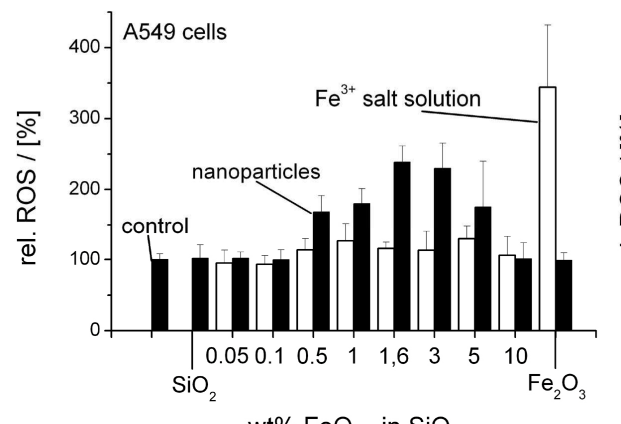

wt\% $\mathrm{FeO}_{1.5}$ in $\mathrm{SiO}_{2}$

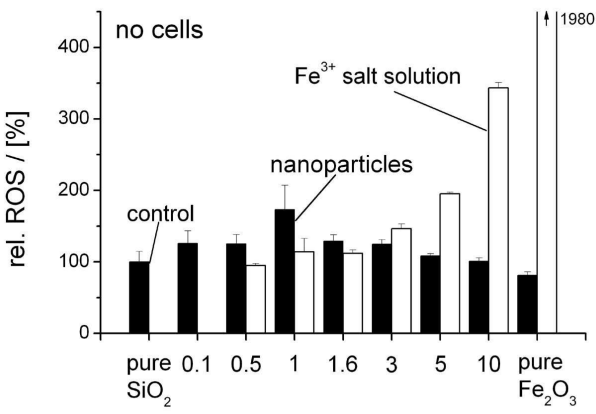

$w t \% \mathrm{FeO}_{1.5}$ in $\mathrm{SiO}_{2}$

ROS production of $30 \mathrm{ppm}$ iron/silica nanoparticles exposed to A549 cells (left) relative to saline controls exactly follows the activity pattern of $\mathrm{FeO} / \mathrm{SiO}_{2}$ known for heterogeneous catalysts. Exposure to nanoparticles (full columns) results indifferent ROS levels than exposure to iron ions (empty bars) at the same concentration. A cell free control experiment in medium (right) showed no statistically relevant dependence of ROS levels on the nanoparticles composition (full columns). Exposure to iron salts at the same iron dose did provoke some ROS above $5 \mathrm{ppm}(\mathrm{Fe}$ ). Much less ROS was formed with A549 cells exposed to $30 \mathrm{ppm}$ aqueous iron (left, empty bar, about $320 \%$ ) if compared to the cell free control (right, empty bar, about $1980 \%$ ) corroborating the barrier function of cell membranes for ions.

\section{Conclusions}

The present investigation has shown that the chemical composition of nanoparticles is a most decisive factor determining the formation of ROS in exposed cells. Beyond mass-based chemical effects where a toxic substance enters and damages a tissue or a cell, the size and mobility of nanoparticles give rise to two other effects: Partially soluble materials such as cobalt oxide may be taken up into cells by a Trojan-horse type mechanism which can significantly increase the damaging action of such materials. Catalytically active nanoparticles can give rise to prolonged damaging action in a cell since the material is not degraded during its interference with intracellular constituents.

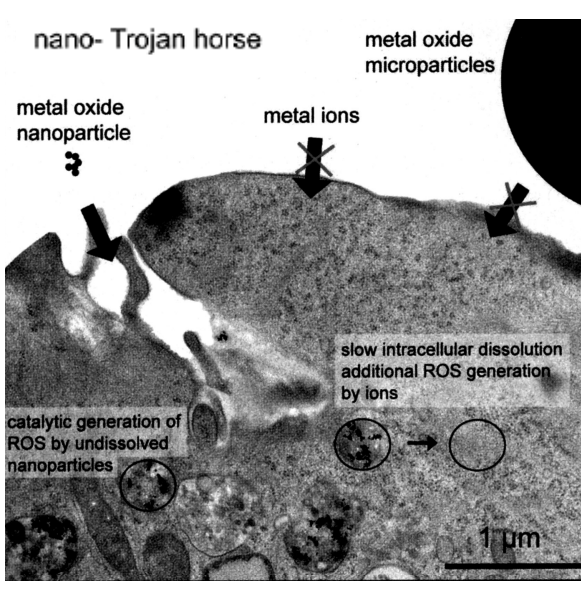

